TAIWANESE JOURNAL OF MATHEMATICS

Vol. 16, No. 5, pp. 1911-1922, October 2012

This paper is available online at http://journal.taiwanmathsoc.org.tw

\title{
ALMOST SELF-CENTERED MEDIAN AND CHORDAL GRAPHS
}

\author{
Kannan Balakrishnan, Boštjan Brešar, Manoj Changat, Sandi Klavžar, \\ Iztok Peterin and Ajitha R. Subhamathi
}

\begin{abstract}
Almost self-centered graphs were recently introduced as the graphs with exactly two non-central vertices. In this paper we characterize almost selfcentered graphs among median graphs and among chordal graphs. In the first case $P_{4}$ and the graphs obtained from hypercubes by attaching to them a single leaf are the only such graphs. Among chordal graph the variety of almost self-centered graph is much richer, despite the fact that their diameter is at most 3 . We also discuss almost self-centered graphs among partial cubes and among $k$-chordal graphs, classes of graphs that generalize median and chordal graphs, respectively. Characterizations of almost self-centered graphs among these two classes seem elusive.
\end{abstract}

\section{INTRODUCTION}

Centrality notions lie in the very center of (discrete) location theory, self-centered graphs $[1,5,14,21]$ forming a prominent theoretical model, see also the survey [4]. Their importance lie in the fact that the maximum eccentricity of any vertex is as small as possible which in turn allows different efficient locations of the emergency facilities at central locations. In some situations, however, we would like to have certain resources not to lie in the center of a graph. With this motivation, almost self-centered graphs were introduced in [17] as the graphs with exactly two non-central vertices. In the seminal paper constructions that produce almost self-centered graphs are described, and embeddings of graphs into smallest almost self-centered graphs are considered. In the present paper we continue these studies by considering almost self-centered graphs among median graphs, chordal graphs, and their generalizations.

Median graphs are probably the most extensively studied class in all metric graph theory. For a survey on median graphs dealing with their characterizations, location theory, and related structures see [16], and for more on these graphs see the book [11]

Received September 23, 2011, accepted February 15, 2012.

Communicated by Gerard Jennhwa Chang.

2010 Mathematics Subject Classification: 05C12, 05C75, 90B80.

Key words and phrases: Radius, Diameter, Almost self-centered graph, Median graph, Chordal graph. 
and recent papers $[6,12]$. Here we only emphasize that despite the fact that median graphs are bipartite, they are intimately connected with triangle-free graphs [13].

Chordal graphs, defined as the graphs having no induced cycles of length greater than 3, are by far the most investigated class of graphs, see e.g. [2]. They have been studied from numerous aspects and generalized in several ways. A very natural generalization are the so-called $k$-chordal graphs, in which by definition the longest induced cycles are of length $k$. The largest common subclass of chordal and median graphs are trees, indicating the tree-like structure of both classes. On the other hand, chordal and median graphs have a common generalization through the so-called cage-amalgamation graphs [3], for which certain tree-like equalities were proven that generalize such equalities in median graphs (counting the numbers of induced hypercubes) and in chordal graphs (counting the numbers of induced cliques).

The paper is organized as follows. In the next section definitions needed and concepts considered are collected. In Section 3 self-centered and almost self-centered median graphs are characterized and related partial cubes are considered. In Section 4 we concentrate on chordal graphs and prove that the diameter of a chordal almost self-centered graph is not more than 3. We follow with a characterization of almost self-centered chordal graph and provide several infinite subclasses of them. In the final section $k$-chordal graphs are considered and proved that the diameter of a $k$-chordal almost self-centered graph with $k \geq 4$ is at most $k$. A characterization of $k$-chordal almost self-centered graphs remains an open problem.

\section{Preliminaries}

The distance considered in this paper is the usual shortest path distance $d$. A shortest path between vertices $u$ and $v$ will also be called a $u, v$-geodesic. The eccentricity $\operatorname{ecc}(v)$ of a vertex $v$ is the distance to a farthest vertex from $v$. A vertex $v$ is said to be an eccentric vertex of $u$ if $d(u, v)=\operatorname{ecc}(v)$. The radius $\operatorname{rad}(G)$ of $G$ and the diameter $\operatorname{diam}(G)$ of $G$ are the minimum and the maximum eccentricity, respectively. A vertex $u$ with $\operatorname{ecc}(u)=\operatorname{rad}(G)$ is called a central vertex, and it is diametrical if $\operatorname{ecc}(u)=\operatorname{diam}(G)$ holds. A graph $G$ is self-centered graph if all vertices are central (equivalently, all vertices are diametrical), and is almost self-centered graph if the center of $G$ consists of $|V(G)|-2$ vertices.

For a connected graph and an edge $x y$ of $G$ we denote

$$
W_{x y}=\{w \in V(G) \mid d(x, w)<d(y, w)\} .
$$

Note that if $G$ is a bipartite graph then $V(G)=W_{a b} \cup W_{b a}$ holds for any edge $a b$. Next, for an edge $x y$ of $G$ let $U_{x y}$ denote the set of vertices that are in $W_{x y}$ and have a neighbor in $W_{y x}$. Sets in a graph that are $U_{x y}$ for some edge $x y$ will be called $U$-sets. Similarly we define $W$-sets. If for some edge $x y, W_{x y}=U_{x y}$, we call the set $U_{x y}$ peripheral set or periphery. 
The Cartesian product $G \square H$ of graphs $G$ and $H$ is the graph with vertex set $V(G) \times V(H)$ where the vertex $(g, h)$ is adjacent to the vertex $\left(g^{\prime}, h^{\prime}\right)$ whenever $g g^{\prime} \in E(G)$ and $h=h^{\prime}$, or $g=g^{\prime}$ and $h h^{\prime} \in E(H)$. The Cartesian product is commutative and associative, the product of $n$ copies of $K_{2}$ is the $n$-dimensional hypercube or $n$-cube $Q_{n}$. With $Q_{n}^{+}$we denote the graph obtained from $Q_{n}$ by attaching a pendant vertex to a vertex of $Q_{n}$, while $Q_{n}^{-}$denotes the graph obtained from $Q_{n}$ by removing one of its vertices. ( $Q_{n}$ is vertex-transitive, hence these two graphs are well-defined.) It is straightforward to see that if $G$ and $H$ are self-centered graphs, then so is $G \square H$.

A (connected) graph $G$ is a median graph if for any three vertices $x, y, z$ there exists a unique vertex that lies in $I(x, y) \cap I(x, z) \cap I(y, z)$. (Here $I(u, v)$ denotes the set of vertices on all $u, v$-geodesics, that is, the interval between $u$ and $v$.) If $u v$ is an edge of a median graph, then the set of edges between $U_{u v}$ and $U_{v u}$ form a matching. Two of the most important classes of median graphs are trees and hypercubes. For the next result see [11, Lemma 12.20]:

Proposition 2.1. Let $G$ be a median graph. Then $G$ is a hypercube if and only if $W_{u v}=U_{u v}$ holds for any edge uv of $G$.

A subgraph $H$ of $G$ is isometric if $d_{H}(u, v)=d_{G}(u, v)$ for all $u, v \in V(H)$ and a graph $G$ is a partial cube if it is an isometric subgraph of some $Q_{n}$, see [11,22]. It is well-known that median graphs are partial cubes but not the other way around.

\section{Almost-self Centered Median Graphs and Partial Cubes}

We begin with the following strengthening of a result of Mulder from [20] asserting that the same conclusion holds provided each vertex of a median graph has a unique diametrical vertex.

Proposition 3.1. Let $G$ be a median graph. Then $G$ is self-centered if and only if $G$ is a hypercube.

Proof. Clearly, hypercubes are self-centered. For the converse it suffices (in view of Proposition 2.1) to prove that $W_{u v}=U_{u v}$ holds for any edge $u v$ of $G$.

Suppose there is a vertex $x$ in $W_{u v}-U_{u v}$ for some edge $u v \in E(G)$. We may assume that $x$ is adjacent to $u$. Note that any eccentric vertex $\bar{u}$ of $u$ lies in $W_{v u}$, for otherwise $\operatorname{ecc}(v)>\operatorname{ecc}(u)$. But then $d(x, \bar{u})=1+d(u, \bar{u})$, a contradiction. Hence $W_{u v}=U_{u v}$ holds.

Theorem 3.2. Let $G$ be a median graph. Then $G$ is almost self-centered if and only if $G$ is either $P_{4}$ or $Q_{n}^{+}$for some $n \geq 1$.

Proof. It is straightforward to see that $P_{4}$ and $Q_{n}^{+}, n \geq 1$, are almost self-centered graphs. 
Let now $G$ be an arbitrary almost self-centered median graph. Since $G$ is not self-centered, Propositions 3.1 and 2.1 imply that there exists an edge $u v \in E(G)$ and a vertex $x \in W_{u v}-U_{u v}$. Select the edge $u v$ and the vertex $x$ such that $x u \in E(G)$. Let $\bar{u}$ be an eccentric vertex of $u$.

Case 1. $\bar{u} \in W_{v u}$.

Then $d(u, \bar{u})=d$ and $d(x, \bar{u})=d+1$, that is, $x$ and $\bar{u}$ are diametrical vertices in this case. We claim that $W_{u v}-U_{u v}=\{x\}$ and suppose on the contrary that there exists $y \in W_{u v}-U_{u v}, y \neq x$. Suppose first that $y$ is adjacent to $y^{\prime} \in U_{u v}$. Let $y^{\prime \prime}$ be the neighbor of $y^{\prime}$ in $U_{v u}$. If an eccentric vertex $\overline{y^{\prime}}$ of $y^{\prime}$ lies in $W_{v u}$, then $d\left(y, \overline{y^{\prime}}\right)=d+1$, a contradiction. Therefore, $\overline{y^{\prime}} \in W_{u v}$. Then $d\left(y^{\prime \prime}, \overline{y^{\prime}}\right)=d+1$ hence $\overline{y^{\prime}}=x$ and $y^{\prime \prime}=\bar{u}$. Then $d(y, v)=d+1$, another contradiction.

Hence $u$ is the only vertex of $U_{u v}$ that has a neighbor in $W_{u v}-U_{u v}$. It is now clear that $x$ is the unique vertex from $W_{u v}-U_{u v}$ for otherwise we would have more than two diametrical vertices or diameter bigger than $d+1$. So we have proved that $W_{u v}-U_{u v}=\{x\}$.

Assume $\bar{u} \in W_{v u}-U_{v u}$. We are going to show that $W_{v u}-U_{v u}=\{\bar{u}\}$. Let $y$ be an arbitrary vertex from $W_{v u}-U_{v u}$ with a neighbor $z \in U_{v u}$. Let $\bar{z}$ be an eccentric vertex of $z$. Then $\bar{z} \in W_{u v}$, for otherwise, the neighbor of $z$ in $U_{u v}$ would have eccentricity $d+1$. Since $d(y, \bar{z})=d+1$, we find that $\bar{z}=x$ and $y=\bar{u}$. We conclude that $W_{v u}-U_{v u}=\{\bar{u}\}$ by the same reasons as above. Let the neighbor of $\bar{u}$ in $U_{v u}$ be $z^{\prime \prime}$ and let $z^{\prime}$ be the neighbor of $z^{\prime \prime}$ in $U_{u v}$. If an eccentric vertex of $z^{\prime}$ lies in $W_{u v}$, then the eccentricity of $\bar{u}$ is $d+2$, which is not possible. So an eccentric vertex $\overline{z^{\prime}}$ of $z^{\prime}$ has to lie in $W_{v u}$. If $\overline{z^{\prime}} \in U_{v u}$, then $\bar{u}$ has an eccentric vertex (the neighbor of $\overline{z^{\prime}}$ in $U_{u v}$ ) different from $x$. Therefore, $\overline{z^{\prime}}=\bar{u}$. This means that $d=2$ and consequently $G=P_{4}$.

Suppose $\bar{u} \in U_{v u}$. By similar arguments as before $W_{v u}=U_{v u}$. Clearly, an eccentric vertex of $y \in U_{u v}$ lies in $U_{v u}$, for any $y$. Since $G-x=\left\langle U_{u v}\right\rangle \square K_{2}$, we also have that an eccentric vertex of $y^{\prime} \in U_{v u}$ lies in $U_{u v}$, for any $y^{\prime}$. Then $G-x$ is a hypercube by Proposition 2.1 and therefore $G=Q_{n}^{+}$.

Case 2. $\bar{u} \in W_{u v}$.

In this case, $\bar{u} \notin U_{u v}$, for otherwise $u$ would have eccentricity $d+1$. By interchanging the roles of $x$ and $v$ we are in Case 1 .

In the rest of the section we consider (almost) self-centered partial cubes. Even cycles form an example of self-centered partial cubes, and we can expect that the list of (almost) self-centered graphs will be considerably larger than for median graphs. However, their characterization seems difficult, just as it is difficult to characterize regular (in particular cubic) partial cubes, see $[9,15,18]$. We give a construction, based on an expansion procedure, that gives rise to new self-centered partial cubes from smaller ones. Again, as for median graphs, they give rise to almost self-centered graphs by adding a pendant vertex. However these are not the only almost self-centered partial cubes as we will see at the end of the section. 
Let $G_{1}$ and $G_{2}$ be isometric subgraphs of a graph $G$ such that $G_{1} \cup G_{2}=G$ and $G^{\prime}=G_{1} \cap G_{2} \neq \emptyset$. Note that there is no edge from $G_{1} \backslash G^{\prime}$ to $G_{2} \backslash G^{\prime}$. Then the expansion of $G$ with respect to $G_{1}$ and $G_{2}$ is the graph $H$ defined as follows. Take disjoint copies of $G_{1}$ and $G_{2}$ and connect every vertex from $G^{\prime}$ in $G_{1}$ with the same vertex of $G^{\prime}$ in $G_{2}$ with an edge. It is not hard to see that copies of $G^{\prime}$ in $G_{1}$ and in $G_{2}$ and new edges between those two copies form the Cartesian product $G^{\prime} \square K_{2}$. Chepoi [7] has shown that $G$ is a partial cube if and only if $G$ can be obtained from $K_{1}$ by a sequence of expansions. Similar expansion theorem was shown for median graphs earlier by Mulder [19].

We call the expansion $H$ of $G$ with respect to $G_{1}$ and $G_{2}$ a diametrical expansion whenever for any diametrical pair of vertices $u$ and $\bar{u}$ of $G$ either both $u, \bar{u} \in V\left(G^{\prime}\right)$ or $u \in V\left(G_{1} \backslash G^{\prime}\right)$ and $\bar{u} \in V\left(G_{2} \backslash G^{\prime}\right)$.

Theorem 3.3. Let $G$ be a self-centered partial cube and let $H$ be obtained from $G$ by a diametrical expansion. Then $H$ is a self-centered partial cube.

Proof. Let $H$ be a diametrical expansion of $G$ with respect to $G_{1}$ and $G_{2}$ where $G^{\prime}=G_{1} \cap G_{2}$. Then $H$ is a partial cube by Chepoi's theorem.

Let $\operatorname{ecc}_{G}(g)=d$ for any $g \in V(G)$. Let $h$ be an arbitrary vertex of $H$. Then $h$ must be in either $G_{1} \backslash G^{\prime}, G_{2} \backslash G^{\prime}$ or $G^{\prime} \square K_{2}$. First we assume that $h \in V\left(G_{1} \backslash G^{\prime}\right)$. Then $\bar{h}$ must be in $G_{2} \backslash G^{\prime}$, since $H$ is a diametrical expansion. Also ecc ${ }_{H}(h)=d+1$. Namely, to see $\operatorname{ecc}_{H}(h) \geq d+1$ we can take the same path as in $G$ between $h$ and $\bar{h}$ that is extended by a new edge of expansion, and in addition $\operatorname{ecc}_{H}(h)>d+1$ would yield a contradiction with $\operatorname{ecc}_{G}(h)=d$. By symmetry we also have $\operatorname{ecc}_{H}(h)=d+1$ for $h \in V\left(G_{2} \backslash G^{\prime}\right)$. Next let $h \in V\left(G^{\prime} \square K_{2}\right)$. Then $h, \bar{h} \in V\left(G^{\prime}\right)$. Let $\overline{h_{1}} \in V\left(G_{1}\right)$ and $\overline{h_{2}} \in V\left(G_{2}\right)$ be two copies of $\bar{h}$ in $H$. If $h \in\left(G_{1}\right)$ then $d_{H}\left(h, \overline{h_{2}}\right)=d+1$ and if $h \in\left(G_{2}\right)$ then $d_{H}\left(h, \overline{h_{1}}\right)=d+1$. Again $\operatorname{ecc}_{H}(h)>d+1$ would yield a contradiction with $\operatorname{ecc}_{G}(h)=d$ and we have $\operatorname{ecc}_{H}(h)=d+1$.

An example of diametrical expansion is shown in Figure 1. Since $Q_{3}$ is a selfcentered partial cube, so is the expanded graph on the right hand side of the figure.

It is easy to see (by induction for instance) that if a partial cube is obtained from $K_{1}$ by a series of diametrical expansions, then every vertex has a unique diametrical vertex. We can obtain almost self-centered partial cubes from self-centered partial cube $G$ by attaching a pendant vertex to a vertex with the unique diametrical vertex in $G$. However this is not the only possibility. Another family of almost self-centered partial cubes arise from $Q_{n}^{-}$by attaching a pendant vertex to a vertex of degree $n-1$. Note that for $n=2$ we get the sporadic example $P_{4}$ of median graphs.

We can generalize the above idea as follows. Let $G$ be a self-centered graph, and let $G_{1}$ and $G_{2}$ be isometric subgraphs of $G$ such that the expansion of $G$ with respect to $G_{1}$ and $G_{2}$ is "almost diametrical", that is, there is exactly one pair $(u, \bar{u})$ of diametrical vertices with the property $u \in V\left(G_{1} \backslash G^{\prime}\right)$ and $\bar{u} \in V\left(G^{\prime}\right)$ and for all other 
diametrical pairs the condition of diametrical expansion holds. Such an expansion does not produce self-centered graphs, but if we attach a pendant vertex to $u$ we get an almost self-centered graph.

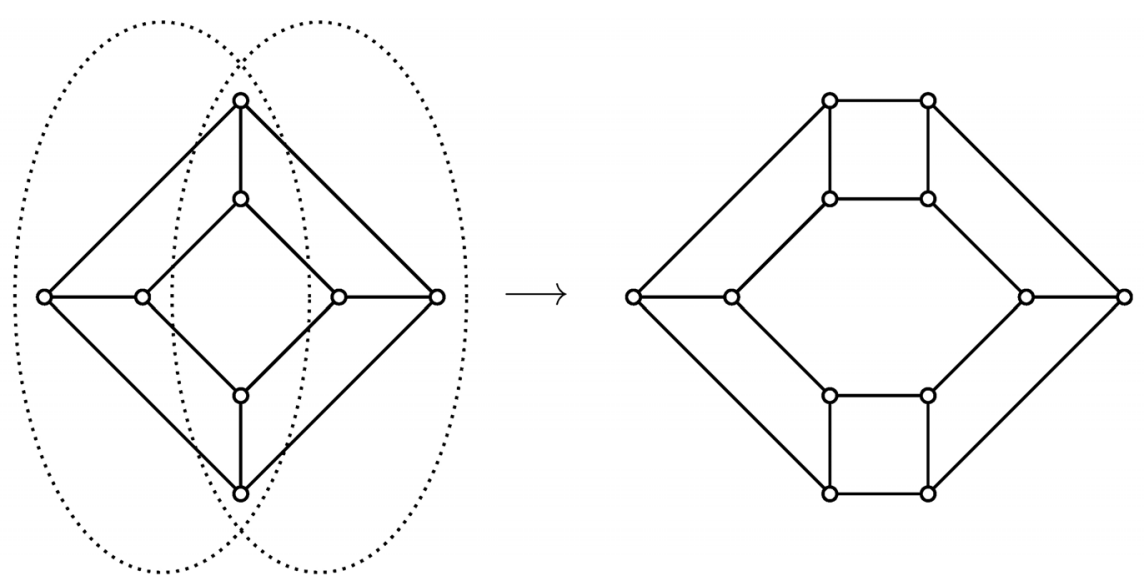

Fig. 1. A diametrical expansion of $Q_{3}$

\section{Chordal Graphs}

In this section we characterize almost self-centered chordal graphs. For this purpose we first show:

Theorem 4.1. Let $G$ be a chordal, almost self-centered graph. Then $\operatorname{diam}(G) \leq 3$.

Proof. Suppose on the contrary that $G$ is a chordal almost self-centered graph with diam $=k \geq 4$. Let $x$ and $\bar{x}$ be diametrical vertices with $\operatorname{ecc}(x)=\operatorname{ecc}(\bar{x})=k$ and let $P:(x=) u_{0} u_{1} \ldots u_{k}(=\bar{x})$ be an $x, \bar{x}$-diametrical path. Then $\operatorname{ecc}(u)=k-1$ for all the other vertices $u \in V(G)-\{x, \bar{x}\}$. Hence there exists $\overline{u_{2}}$ with $d\left(u_{2}, \overline{u_{2}}\right)=k-1$.

Since $d\left(u_{2}, \overline{u_{2}}\right)=k-1$, we have $k-3 \leq d\left(x, \overline{u_{2}}\right) \leq k-1$. Let $Q:(x=$ ) $v_{0} v_{1} \ldots v_{q}\left(=\overline{u_{2}}\right), k-3 \leq q \leq k-1$, be a shortest $x, \overline{u_{2}}$-path. Note that it is possible that $v_{1}=u_{1}$, but all other vertices of $P$ and $Q$ are different. For if a vertex $u_{s}$, where $s \geq 2$, belongs to both $P$ and $Q$, then $u_{2}$ is an inner vertex on a $x, \overline{u_{2}}$-geodesic of length at most $k-1$, a contradiction with $d\left(u_{2}, \overline{u_{2}}\right)=k-1$.

Let $R:(\bar{x}=) w_{0} w_{1} \ldots w_{r}\left(=\overline{u_{2}}\right), 2 \leq r \leq k-1$, be a $\overline{u_{2}}, \bar{x}$-geodesic (note that the case when $\bar{x}$ and $\overline{u_{2}}$ are adjacent is not excluded). Let $v_{j}$ and $u_{i}$ be the first vertices of $Q$ and $P$, respectively, that are also on $R$. Suppose $u_{1} \neq v_{1}$. The $x, u_{i}$-subpath of $P$, the $x, v_{j}$-subpath of $Q$, and the $u_{i}, v_{j}$-subpath of $R$ form a cycle $C$. Clearly, $u_{2}$ does not form a chord with any vertex of $P, Q$, and $R$ since $d\left(u_{2}, \overline{u_{2}}\right)=k-1$, with a possible exception of $v_{1}$ and $w_{1}$. The later case is possible only when $d\left(\bar{x}, \overline{u_{2}}\right)=k-1$, since otherwise $d\left(u_{2}, \overline{u_{2}}\right)<k-1$ which is not possible. But then clearly $d(x, \bar{x})=4$. 
Let first $k>4$. If $u_{2} v_{1}$ is not a chord, then $u_{2}$ has no chords on $C$ and we are done since also $u_{1} u_{3}$ is not a chord. So we may assume that $u_{2} v_{1}$ is a chord (which also includes the case when $u_{1}=v_{1}$ ). Then let $C^{\prime}$ be a cycle obtained from $u_{2} v_{1}$ and a longer $u_{2}, v_{1}$-path on $C$. Then $u_{2}$ has no chords on $C^{\prime}$ and $u_{3}$ and $v_{1}$ are not adjacent since $d(x, \bar{x})=k$. Hence the same contradiction again.

Finally let $k=4$. If $u_{2} w_{1}$ is not a chord we have the same contradiction as before. So let $u_{2} w_{1} \in E(G)$ and let $C^{\prime}$ be a cycle obtained from $u_{2}, w_{1}$-path on $C$ that does not contains $\bar{x}$ and $u_{2} w_{1}$. If $u_{2} v_{1} \notin E(G)$ no chord on $C^{\prime}$ starts in $u_{2}$. We get a contradiction, since edge $u_{1} w_{1}$ would destroy $d(x, \bar{x})=4$. Hence $u_{2} v_{1} \in E(G)$ and let $C^{\prime \prime}$ be a cycle obtained from edge $u_{2} v_{1}$ and $u_{2}, v_{1}$-path on $C^{\prime}$ that does not contains $u_{1}$. (Note that if $u_{1}=v_{1}$ we have $C^{\prime \prime}=C^{\prime}$.) But again $u_{2}$ has no chords on $C^{\prime \prime}$ and $v_{1} w_{1}$ is again not possible since $d(x, \bar{x})=4$, a final contradiction.

We introduce the class $\mathcal{C}$ of chordal graphs as follows. Let $G^{\prime}$ be a chordal graph with diameter at most 2, and let $V\left(G^{\prime}\right)=X+Y+Z$ (where + stands for the disjoint union of sets) such that for any $v \in V\left(G^{\prime}\right)$, we have $d(v, X) \leq 1$ and $d(v, Y) \leq 1$, with only $Z$ being possibly empty. (Note that it means any vertex from $X$ must have a neighbor in $Y$ and vice versa and a vertex in $Z$, if any, must have a neighbor in both $X$ and $Y$.) Let $G$ be obtained from $G^{\prime}$ by adding two new vertices $x$ and $y$, and edges between $x$ and all vertices from $X$, and $y$ and all vertices from $Y$. Clearly, any graph $G$, obtained in such a way is chordal with diameter 3 , and we say it belongs to the class $\mathcal{C}$. It is also clear that only $x$ and $y$ are diametrical vertices, and all other vertices have the same eccentricity, making $G$ almost self-centered chordal graph.

The class $\mathcal{C}$ is relatively rich. It includes the graphs, obtained from a clique by adding two vertices with disjoint neighborhoods in the clique (say, $P_{4}$ as the smallest example. Another subclass is obtained from the join $K_{n} \circ \overline{K_{m}}$ of the complete graph $K_{n}$ and totally disconnected graph $\overline{K_{m}}$, by adding two simplicial vertices, whose neighborhoods are disjoint subcliques of $K_{n}$.

Theorem 4.2. Let $G$ be a chordal graph. Then $G$ is almost self-centered if and only if $G$ is either $K_{n}-e$ or it belongs to $\mathcal{C}$.

Proof. If $G$ is either $K_{n}-e$ or in $\mathcal{C}$, it is clearly almost self-centered.

For the converse, let $G$ be an almost self-centered chordal graph. By Theorem 4.1, $G$ has diameter at most 3. Assume $\operatorname{diam}(G)=2$. Since $G$ is not a complete graph, there exist two non adjacent simplicial vertices $x$ and $y$ in $G$ by Dirac's theorem [8]. Clearly $N(x) \cap N(y) \neq \emptyset$. In addition, if there is a vertex $z \notin N[x] \cap N[y]$ then $\operatorname{ecc}(z)=2$ which is a contradiction with $G$ being almost self-centered. By the same reasoning, we find that $N(x)=N(y)$ induces a clique. Hence $G$ is isomorphic to $K_{n}-e$.

Suppose $\operatorname{diam}(G)=3$. Then by a result of Farber and Jamison [10] there exist two simplicial vertices $x$ and $y$ with $d(x, y)=\operatorname{diam}(G)=3$. Let $X=N(x)$ and 
$Y=N(y)$. Since $d(x, y)=3, X \cap Y=\emptyset$ and let $Z=V(G)-(N[x] \cup N[y])$. Clearly, the subgraph $G^{\prime}$ induced by $V(G)-\{x, y\}$ is chordal and its diameter is at most 2. Note that for any $v \in V\left(G^{\prime}\right), \operatorname{ecc}_{G}(v)=2$. If $v \in X$ then there must be a vertex $w \in Y$ such that $v w \in E(G)$, otherwise $d(v, y)=3$. We infer that $d(v, Y)=1$, and similarly we find that $d(w, X)=1$ for any $w \in Y$. If $z \in Z$, again by eccentricity 2 of vertices from $G^{\prime}$, we find that $d(z, X)=1$ and $d(z, Y)=1$. We derive that $G$ belongs to the class $\mathcal{C}$.

\section{5. $k$-CHORDAL GRAPHS}

A graph $G$ is $k$-chordal if every cycle $C$ of length greater than $k$ has a chord. The chordality of $G$ is the smallest $k$ such that $G$ is $k$-chordal. For $k$-chordal graphs Theorem 4.1 naturally extends:

Theorem 5.1. Let $G$ be a $k$-chordal almost self-centered graph with $k \geq 4$. Then $\operatorname{diam}(G) \leq k$.

Proof. Suppose on the contrary that $G$ is a $k$-chordal almost self-centered graph with $\operatorname{diam}(\mathrm{G})=r \geq k+1$. Let $x$ and $\bar{x}$ be diametrical vertices with $\operatorname{ecc}(x)=$ $\operatorname{ecc}(\bar{x})=r$ and let $P:(x=) u_{0} u_{1} \ldots u_{r}(=\bar{x})$ be a $x, \bar{x}$-diametrical path. Then $\operatorname{ecc}(u)=r-1$ for all the other vertices $u \in V(G)-\{x, \bar{x}\}$. For $a=\left\lceil\frac{k-1}{2}\right\rceil+1$ there exists $\overline{u_{a}}$ with $d\left(u_{a}, \overline{u_{a}}\right)=r-1$.

Since $d\left(u_{a}, \overline{u_{a}}\right)=r-1$, we have $r-a-1 \leq d\left(x, \overline{u_{a}}\right) \leq r-1$. Let $Q:(x=$ ) $v_{0} v_{1} \ldots v_{q}\left(=\overline{u_{k-1}}\right), r-a-1 \leq q \leq r-1$, be a shortest $x, \overline{u_{a}}$-path. Note that it is possible that $v_{i}=u_{i}$ for $1 \leq i<a$, but all other vertices of $P$ and $Q$ are different. For if a vertex $u_{s}, s \geq a$, belongs to both $P$ and $Q$, then $u_{a}$ is an inner vertex on a shortest $x, \overline{u_{a}}$-path of length at most $r-1$, a contradiction with $d\left(u_{a}, \overline{u_{a}}\right)=r-1$.

Let $R:(\bar{x}=) w_{0} w_{1} \ldots w_{t}\left(=\overline{u_{a}}\right), 1 \leq t \leq r-1$ be a shortest $\overline{u_{a}}, \bar{x}$-path. Let $u_{\ell}$ be the last vertex common to $P$ and $Q$, while $v_{p}$ and $u_{s}=w_{r-s}$ be the first vertices of $Q$ and $P$, respectively, that are also on $R$. Suppose that there exists a chord $u_{a} v_{b}$ for some $\ell \leq b \leq s$. Then $q-b+1 \geq d\left(u_{a}, \overline{u_{a}}\right)=r-1$ and hence $b \leq q-r+2 \leq r-1-r+2=1$. Clearly $u_{a} v_{0}=u_{a} x \notin E(G)$ and $u_{a} v_{1} \in E(G)$ imply a contradiction with $d(x, \bar{x})=r \geq k+1$. Hence there is no chord $u_{a} v_{b}$. Similarly if there exists a chord $u_{a} w_{b}$ for some $r-s \leq b \leq p$ we have $t-b+1 \geq r-1$ and again $b \leq 1$. As before edge $u_{a} w_{0}$ is not possible, but edge $u_{a} w_{1}$ can exists when $r=5$ and $k=4$. For $r>5$ this is not possible since we violate $d(x, \bar{x})=r$.

Assume first that $u_{a} w_{1} \notin E(G)$. Fix edges $u_{b} v_{c}, u_{d} w_{e}$, and $v_{f} w_{g}, f \geq c, g \geq e$, and $b<a<d$, as follows. Let $b<a$ be the biggest number with an edge $u_{b} v_{y}$ and among all such edges let $c$ also be the biggest number. Note that such an edge always exists, since $u_{\ell+1} v_{\ell}$ is such an edge. Similarly let $d>a$ be smallest number with an edge $u_{d} w_{y}$ and among all such edges choose $e$ to be the biggest number. Again such an edge exists since $u_{d-1} u_{d}$ is of that type. Finally let $f \geq c$ be the smallest number 
such that edge $v_{f} w_{g}$ exists, where $g \geq e$ is also small as possible. Since $v_{p-1} v_{p}$ is a candidate for this edge, there is no problem with the existence of such an edge. We construct cycle $C$ as follows

$$
u_{b} v_{c} \stackrel{Q}{\rightarrow} v_{f} w_{g} \stackrel{R}{\rightarrow} w_{e} u_{d} \stackrel{P}{\rightarrow} u_{b} .
$$

By minimality or maximality of $b, c, d, e, f, g$ it is clear that $C$ is chordless. We gain the contradiction by showing that $C$ has length $>k$.

First note that since $d(x, \bar{x})=r, v_{f}$ is not adjacent to $w_{g}$ for $g<r-f-1$. Thus $g \geq r-f-1$. Clearly

$$
|C|=d-b+f-c+g-e+3 \geq r+2+d-b-c-e .
$$

We will show that $2+d-b-c-e \geq 0$ or equivalently $d-b+2 \geq c+e$. Let $P^{\prime}$ be a path $u_{a} \stackrel{P}{\rightarrow} u_{d} w_{e}$ and $P^{\prime \prime}$ a path $u_{a} \stackrel{P}{\rightarrow} u_{b} v_{c}$. Note that $P^{\prime} \cap P^{\prime \prime}=\left\{u_{a}\right\}$ and that the length of both is $d-b+2$. If $c>\left|P^{\prime \prime}\right|$ we have $q=d\left(x, \overline{u_{a}}\right)>\left|P^{\prime \prime}\right|+q-c \geq r-1$, a contradiction. Similarly if $e>\left|P^{\prime}\right|$ we have $t=d\left(\bar{x}, \overline{u_{a}}\right)>\left|P^{\prime}\right|+t-e \geq r-1$, a contradiction again. Thus $c \leq\left|P^{\prime \prime}\right|$ and $e \leq\left|P^{\prime}\right|$ and $d-b+2 \geq c+e$ follows. Hence $C$ is a chordless cycle of length $|C| \geq r+2+d-b-c-e \geq r>k$ which is not possible in $k$-chordal graphs.

Finally let $u_{a} w_{1} \in E(G)$. Then $r=5, k=4$, and in this case $a=3$. Since $d\left(u_{3}, \overline{u_{3}}\right)=4$ it is easy to see that $u_{2} w_{2}, u_{2} w_{1}, u_{1} w_{1}$, and $u_{1} w_{0}$ are all possible edges of type $u_{b} v_{c}$. Instead of $u_{d} w_{e}$ we take $u_{3} w_{1}$ and for edge $v_{f} w_{g}$ we have the following possibilities: $v_{1} w_{3}, v_{2} w_{2}, v_{2} w_{3}, v_{3} w_{2}$, and $v_{3} w_{3}$ (whenever this vertices exists). It is easy to see that combining this edges we always get a chordless cycle of length at least 5 , which in not possible in 4-chordal graphs.

As a direct consequence of Theorem 5.1 we get:

Corollary 5.2. If $G$ is an almost self-centered graph of chordality $k$, then $\operatorname{diam}(G)$ $\leq k$.

\section{ACKNOWLEDGMENTS}

Work supported by the Ministry of Science of Slovenia and by the Ministry of Science and Technology of India under the bilateral India-Slovenia grants BI-IN/10-12001 and INT/SLOVENIA-P-17/2009, respectively and by the Research Grants P1-0297 of Ministry of Higher Education, Science and Technology Slovenia and 2/48(2)/2010/ NBHM-R and D by NBHM/DAE, India. B. B., S. K. and I. P. are also with the Institute of Mathematics, Physics and Mechanics, Jadranska 19, 1000 Ljubljana.

\section{REFERENCES}

1. J. Akiyama, K. Ando and D. Avis, Miscellaneous properties of equi-eccentric graphs, in: Convexity and graph theory (Jerusalem, 1981), Vol. 87, NorthHolland Math. Stud., North-Holland, Amsterdam, 1984, pp. 13-23. 
2. A. Brandstädt, V. B. Le and J. P. Spinrad, Graph Classes: A Survey, SIAM, Philadelphia, PA, 1999.

3. B. Brešar and A. Tepeh Horvat, Cage-amalgamation graphs, a common generalization of chordal and median graphs, European J. Combin., 30 (2009), 10711081.

4. F. Buckley, Self-centered graphs, in: Graph theory and its applications: East and West (Jinan, 1986), Vol. 576, Ann. New York Acad. Sci., New York Acad. Sci., New York, 1989, pp. 71-78.

5. F. Buckley, Z. Miller and P. J. Slater, On graphs containing a given graph as center, J. Graph Theory, 5(4) (1981), 427-434.

6. C. T. Cheng, A poset-based approach to embedding median graphs in hypercubes and lattices, Order, 29 (2012), 147-163.

7. V. Chepoi, Isometric subgraphs of Hamming graphs and d-convexity, Kibernetika (Kiev), 1 (1988), 6-10.

8. G. Dirac, On rigid-circuit graphs, Abh. Math. Sem. Univ. Hamburg, 25 (1961), 71-76.

9. D. Eppstein, Cubic partial cubes from simplicial arrangements, Electron. $J$. Combin., 13(1) Research paper 79, 14 pp., 2006.

10. M. Farber and R. E. Jamison, Convexity in graphs and hypergraphs, SIAM J. Alg. Discrete Methods, 7 (1986), 433-444.

11. R. Hammack, W. Imrich and S. Klavžar, Handbook of Product Graphs, 2nd ed., CRC Press, Boca Raton, 2011.

12. W. Imrich and S. Klavžar, Two-ended regular median graphs, Discrete Math., 311 (2011), 1418-1422.

13. W. Imrich, S. Klavžar and H. M. Mulder, Mediangraphs and triangle-free graphs, SIAM J. Discrete Math., 12 (1999), 111-118.

14. T. N. Janakiraman, On self-centered graphs, J. Ramanujan Math. Soc., 7(1) (1992), 83-92.

15. S. Klavžar, Hunting for cubic partial cubes, in: Convexity in Discrete Structures, Vol. 5, Ramanujan Math. Soc. Lect. Notes Ser., Ramanujan Mathematical Society, Mysore, India, 2008, pp. 87-95.

16. S. Klavžar and H. M. Mulder, Median graphs: characterizations, loca-tion theory and related structures, J. Combin. Math. Combin. Comput., 30 (1999), 103-127.

17. S. Klavžar, K. P. Narayankar and H. B. Walikar, Almost self-centered graphs, Acta Math. Sin. (Engl. Ser.), 27 (2011), 2343-2350. 
18. S. Klavžar and S. Shpectorov, Tribes of cubic partial cubes, Discrete Math. Theor. Comput. Sci., 9(1) (2007), 273-291.

19. H. M. Mulder, The structure of median graphs, Discrete Math., 24(2) (1978), 197-204.

20. M. Mulder, $n$-cubes and median graphs, J. Graph Theory, 4(1) (1980), 107-110.

21. S. Negami and G. H. Xu. Locally geodesic cycles in 2-self-centered graphs, Discrete Math., 58(3) (1986), 263-268.

22. S. Ovchinnikov, Partial cubes: structures, characterizations, and constructions, Discrete Math., 308(23) (2008), 5597-5621.

Kannan Balakrishnan

Department of Computer Applications

Cochin University of Science and Technology

Cochin-22, India

E-mail: bkannan@cusat.ac.in

Boštjan Bresar

Faculty of Natural Sciences and Mathematics

University of Maribor

Slovenia

E-mail: bostjan.bresar@uni-mb.si

Manoj Changat

Department of Futures Studies

University of Kerala

Trivandrum-695034

India

E-mail: mchangat@gmail.com

Sandi Klavžar

Faculty of Mathematics and Physics

University of Ljubljana

Slovenia

and

Faculty of Natural Sciences and Mathematics

University of Maribor

Slovenia

E-mail: sandi.klavzar@fmf.uni-lj.si 
Iztok Peterin

Institute of Mathematics and Physics

FEECS University of Maribor

Slovenia

E-mail: iztok.peterin@uni-mb.si

\author{
Ajitha R. Subhamathi \\ Department of Computer Applications \\ N.S.S College \\ Rajakumari, Idukki \\ Kerala, India \\ E-mail: ar.subhamathi@gmail.com
}

